\title{
CHALLENGES OF SCALING AGILE AT ORGANIZATION LEVEL
}

\author{
Florin DUMITRIU \\ Department of Business Information Systems, Faculty of Economics and Business \\ Administration, Alexandru Ioan Cuza University of Iasi \\ fdumi@uaic.ro \\ Gabriela MEȘNIȚĂ \\ Department of Business Information Systems, Faculty of Economics and Business \\ Administration, Alexandru Ioan Cuza University of Iasi \\ gabriela.mesnita@feaa.uaic.ro \\ Dumitru OPREA \\ Department of Business Information Systems, Faculty of Economics and Business \\ Administration, Alexandru Ioan Cuza University of Iasi \\ doprea@uaic.ro \\ Laura-Diana RADU \\ Department of Business Information Systems, Faculty of Economics and Business \\ Administration, Alexandru Ioan Cuza University of Iasi \\ glaura@uaic.ro
}

\begin{abstract}
Large-scale agile development today receives widespread interest in the research and practice areas. In despite of various expressions used in literature for "large-scale" term, two different dimensions could be considered in the context of applying agile methodology: individual level, like project, software or team, and organization level. The aim of this paper is to find out the challenges that are relevant for applying large-scale agile at organization level in order to create agility beyond individual team/project. We found 12 challenges and grouped them in three categories.
\end{abstract}

Keywords: agile methods, large-organizations, challenges

JEL classification: L86, M15, O3

DOI: $10.12948 / \mathrm{ie} 2019.05 .06$

\section{Introduction}

Over the past 15 years, agile software development has increasingly become the norm in the IT industry. Even though the initial fundamental assumptions underpinning agile approach suggested that it is best suited to small projects and single, small, co-located development teams, the agile methods are increasingly applied in large contexts, in projects with tens of teams and hundreds of developers [1].

A clear categorization of the existing streams of research is difficult to recognize [2] due to the lack of general agreement to the actual definition of large-scale in agile software development. Various expressions have been used in the agile literature to denote the large-scale term. Some researchers and practitioners have used general terms, like "large-scale settings" or "large contexts", while others have employed one or even many more specific phrases such "large projects", "large teams", "large, complex software", "large organization" or "mature organization" [3]. Another definition perceives the "largeness" in terms of the complexity of the various knowledge boundaries across the actors and technologies involved [4]. 
If we look at all these phrases, it can be easily noticed that two different dimensions could be considered for the term "large-scale" in the context of applying agile methodology: individual level, like project, software or team, and organization level. Only a few papers have explicitly assumed an organization perspective when analyzing agile adoption at large $[5,6,7]$.

In our paper we are using an organization level perspective and summarizing the literature on the field of agile at large-scale research. Our objective is to find out the challenges that are relevant for applying large-scale agile at organization level in order to create agility beyond individual team/project.

The rest of the paper is organized as follows. In Section 2, we present the research method. In Section 3, we discuss the literature on the topic of company-wide adoption of agile methods, while in Discussion Section we analyze in more details the main challenges and some solutions proposed in literature. Finally, Section 6 concludes the article.

\section{Research methodology}

In this section, we present the result of the literature review on the topic of applying agile in large organizations with an organization level perspective. Our research questions are:

RQ1: What challenges have been reported for applying large-scale agile at the organization level?

RQ2: How could these challenges be grouped for a better understanding?

The research process consisted of four main steps. In the first step we selected a list of papers that contain in their title the relevant expressions for large-scale agile, such as "agile in large settings”, „large scale agile”, ,, agile at large”, „scaling agile”. In the next step we have excluded those papers that contained in their titles expressions relevant for individual levels, like "team", "project", or "software". For the rest of the papers we read keywords and abstracts, and eliminated the papers about organizational agility with a managerial perspective, and those on applying agile in distributed environments as they were focused on specific issues for geographically distributed teams. In the last step, we deeply analyzed the remaining papers to extract the ideas relevant for applying agile in large organizations. In this respect we considered issues like „multi-team environment”, „,enterprise architecture”, „organizational change”.

\section{Literature review}

Organizations try to adopt agile practices to become more competitive, improve processes in order to manage changing requirements, but they face additional challenges in the integration of agile development at the organization level. Although many companies use agile methods, it is still unclear in which environments and under what conditions they really work [8]. Some researchers have stated that agile development in its exactly form is likely not a good solution for large, traditional, top-down systems development organizations [9], [10].

The number and the categorizations of challenges vary from article to article, depending on the research methodology used, the goal of the article and the time in which the research was conducted. The complexity of the agile at large scale approach results from the 79 challenges identified and grouped into 11 categories proposed by Uludag et al. [11]. On the other side, Hobbs and Petit [12] use two broad groups of challenges. First category includes challenges related to the methods themselves, "because of the fixed rule bases and assumptions built into the methods". The second category consists in challenges induced by the enterprise that "will prevent the successful application of the new methods".

We have focused to those challenges of agile adoption that arose at the organizational level in large organizations. The main challenges and their grouping are outlined in Table 1.

The first category includes the challenges that arise in multi-team/multi-project environment specific for large organizations. One of the most prominent challenges for applying agile at 
large is the difficulty in coordinating the work of several agile teams in large organizations [9], [10] and [4]. The team is just one step in an organization's value creation process, and each team has to coordinate their work with other teams. It is quite difficult to bring agility beyond the team as the introduction of agile might create flexibility at the team level, but the surrounding organization could not be responsive enough [6]. Another challenge in multi-team environments is related to the problems that arise from the self-organizing team principle [9], [6], [4] and [12], one of the fundamental assumptions in agile approach.

Table 1. Challenges of agile adoption in large organizations

\begin{tabular}{|c|c|c|}
\hline Category & Challenge & Source \\
\hline \multirow{4}{*}{$\begin{array}{l}\text { Multi- } \\
\text { team/multi- } \\
\text { project } \\
\text { environment }\end{array}$} & Implementing self-organizing teams & {$[6],[4],[12],[9]$} \\
\hline & Coordination of several agile teams & {$[6],[9],[10],[4]$} \\
\hline & Different interpretations of among agile teams & {$[13]$} \\
\hline & $\begin{array}{l}\text { Managing and sharing knowledge with } \\
\text { stakeholders }\end{array}$ & {$[11]$} \\
\hline \multirow{5}{*}{$\begin{array}{l}\text { Organizational } \\
\text { transformation/ } \\
\text { change }\end{array}$} & Management change & {$[10],[6]$} \\
\hline & Culture and leadership behavior change & {$[14]$} \\
\hline & Specialized knowledge in silos & {$[10],[6]$} \\
\hline & Integrating non-development functions & {$[10],[6]$} \\
\hline & $\begin{array}{l}\text { Integration of agile projects with the project } \\
\text { environment's existing processes }\end{array}$ & {$[12],[8]$} \\
\hline \multirow{3}{*}{$\begin{array}{l}\text { Alignment of } \\
\text { individual } \\
\text { projects to } \\
\text { enterprise } \\
\text { business goals }\end{array}$} & $\begin{array}{l}\text { Conflicts between agile projects and holistic } \\
\text { enterprise architecture (EA) }\end{array}$ & [9], [15] \\
\hline & $\begin{array}{l}\text { Integration of EA frameworks and agile } \\
\text { development }\end{array}$ & {$[16],[15]$} \\
\hline & $\begin{array}{l}\text { Balancing between the agility of individual } \\
\text { projects and the organization agility }\end{array}$ & {$[17]$} \\
\hline
\end{tabular}

Large organizations involve numerous people in many development teams. This situation may lead to a lack of alignment between teams regarding the use and implementation of agile practices [13]. Another issue related to multi-team environment is the management and sharing knowledge with stakeholders. These differences lead to some diverging views of knowledge sharing and transfer on agile projects [11]. While agile teams spend time to transfer the information internally, they share few information with external groups.

The second category of challenges encompasses the organization change/transformation. Dikert et al. (cited in [6]) presented three challenges that we consider relevant for applying agile at organizational level: cultural change in organization management, particularly on the middle management level because its role is unclear in agile methods; the difficulty of relocating the people due to the existence of internal boundaries or specialized knowledge in silos; the integration of non-development functions in organizations.

Integration of agile projects with the project environment's existing processes is also an important challenge for large organizations according to the results of a study made by Lindvall et al. [8]. Also, some empirical studies of large-scale agile have indicated challenges related to lack of strategic investments and the inability to change culture and leadership behavior [14]. The last category includes the challenges regarding the alignment of individual projects to the enterprise business goals. Especially in large organizations there is a need to add some structure 
in business processes and the concept of enterprise architecture (EA) is put in place. Some researches stated that the need for a holistic EA when applying agile development is often overlooked [15] and may lead to several problems such as technical debt, unnecessary re-work, inconsistent communication, locally focused isolated design [9], fragile architecture, and divergence in coding style [6]. This situation creates the challenges of integrating of EA frameworks with agile methods and of balancing between projects and organization agility [15], [16] and [17].

\section{Discussion}

Based on literature review we identified two dimensions for agile-at-scale research: at individual level and organizational one. The most papers do not approach these different levels. They present a general view on the agile methods. The results of our literature review highlight 12 challenges which cover the RQ1. They are relevant for applying large-scale agile at organization level. These were grouped into three categories in order to clarify the impact on agile large scale beyond individual team/project. This answers to the RQ2.

Next, in this section, we discuss in more details the main challenges mentioned in the previous section. We also present some solutions to these challenges.

Working in multi-team environment is probably the most common challenge cited in the literature. Large organizations naturally have many interdependencies which require a large amount of coordination that could be hard to manage through mutual adjustment as in agile methods [9]. Moreover, some authors [6] have mentioned some technical consistency problems relating to inter-team coordination, such as integrating the products of teams, lack of standardized build scripts, and synchronizing the definitions of software interfaces between teams. All these create the need for more formal communication with the following main consequences: additional developer overhead and decreasing each project's agility [8]. A solution in this case lies in minimizing the need for cross-team communication and coordination which could be achieved by dividing the work of a large project into independent components called services, like in SOA [9]. Another solution consists in applying one of the available frameworks for scaling agile methods for large context as Scaled Agile Framework (SAFe), Large Scale Scrum (LeSS), Nexus or Disciplined Agile (DAD).

The existence of different agile cultures from team to team makes difficult to transfer people between teams [6]. The application of hybrid methods - agile practices integrated with the traditional ones can solve this problem. They are appropriate when there are mutual dependencies and agile methods are not feasible. In most cases, the organizational transformation proceeded gradually, and during the process it was possible when the new agile method was used in parallel with the old methods [6].

Karvonen et al. [14] consider that it is important to focus on challenges related to the inability to change culture and leadership behavior. As the authors stated, the agile integration is associated with the 'adhocracy' culture oriented toward creativity, innovation effectiveness. Even the management literature designs the cultural profiles for agile integration, there is no single ideal profile, since organizations require different cultural behaviors. For example, agile integration is ineffective in organizations with values such as "secrecy, isolation, complexity, timidity, and disrespect" and could generate many problems rather than benefits. As result, in agile transformation is important to understand the organizational culture.

In the case of using agile methods, organization functions ranging from marketing and sales to human resources have to be integrated in the agile environment, otherwise this might cause serious limitations for the agile implementation. In [11] two issues of organizational changes are highlighted from the perspective of the relationship with the projects beneficiaries (internal and external projects). The internal ones would transform the entire organization, and the 
external ones supposed to redefine the roles of other departments and the relationships with customers, in what might become "agile end-to-end".

Enterprise architecture (EA) is a well-defined practice for conducting enterprise analysis, design, planning, and implementation using a holistic approach in order to guide organizations through the business, information, process, and technology changes, necessary to execute their strategies. The holistic approach of EA and the structure established by its implementation might hinder project progress in a typical agile environment. In order to integrate EA frameworks with agile methods, architects can start documenting standards and guidelines in collaboration with the various agile teams to create a catalogue of standards that all teams have to use [10]. The most of large enterprises use some frameworks, such as COBIT, ITIL, and TOGAF in the regard of ensuring the alignment of IT with overall business goals, but their implementation creates new challenges for applying agile in large organizations. Architecture governance frameworks may conflict with agile development [9] as these frameworks are not tuned to agile teams [15]. Ensuring the compliance of individual agile projects with the EA is an essential aspect of architecture governance and for this reason the organizations must find the best way to approach EA capability for supporting agile development. Also, customizing the agile approach and practices was often seen as a necessary step in the agile implementation.

\section{Conclusions}

Large-scale agile development receives widespread interest in the research and practice areas, although the initial fundamental assumptions suggested that agile approach is best suited to small projects and single, small, co-located development teams. A classification of the existing research is difficult to identify due to the lack of general agreement to the terms used in agile at large scale area. Our literature analysis reveals two different research streams: individual level, like project, software or team, and organization level.

In our paper we placed at organization level with the objective to find out the challenges that are relevant for enterprise-wide agile implementation in large organizations. We grouped the challenges identified in literature in three main categories: multi-team/multi-project environment (implementing self-organizing teams, coordination of several agile teams, different interpretations of agile between teams, managing and sharing knowledge with stakeholders), organizational transformation/change (management change, culture and leadership behavior change, specialized knowledge in silos, integrating non-development functions, integration of agile projects with the project environment's existing processes) and alignment of individual projects to enterprise business goals (conflicts between agile projects and holistic enterprise architecture, integration of EA frameworks and agile development, balancing between the agility of individual projects and the organization agility). The most cited challenge in literature seems to be team's coordination and the most problematic agile characteristic when applying agile in large is self-organizing team.

Our paper can be used by academics as a roadmap for future research and by practitioners to understand and address the challenges of agile methods adoption in wide enterprises and to prepare better the transition from the traditional practices to agile.

\section{References}

[1] M. Jørgensen, "Do agile methods work for large software projects?," in Proc. The International Conference on Agile Software Development, Porto, Portugal, 2018, pp. 179190.

[2] T. Dreesen and P. Diegmann, "Journey Towards Agility: Where Are We Now And Where Are We Heading?," in Proc. The International Conference on Information Systems, Seoul, South Korea, 2017, pp. 1-11. 
[3] F. Dumitriu, G. Meșniță, D. Oprea and L. Radu, "The Need for a Framework in Analyzing the Applying Agile at Large-Scale," in Proc. The 17th International Conference on Informatics in Economy (IE 2017), Iasi, Romania, 2017, pp. 417-424.

[4] K. Rolland, T. Dingsoyr, B. Fitzgerald and K. Stol, "Problematizing agile in the large: alternative assumptions for large-scale agile development," in Proc. The 39th International Conference on Information Systems, Dublin, Irland, 2016, pp. 1-20.

[5] R. Wendler, "Dimensions of Organizational Agility in the Software and IT Service Industry: Insights from an Empirical Investigation," Communications of the Association for Information Systems, vol. 39, no. 1, paper 21, 2016.

[6] M. Paasivaara and C. Lassenius, "Challenges and success factors for large-scale agile transformations: A research proposal and a pilot study," in Proc. The Scientific Workshop Proceedings of XP2016, 2016, Article No. 9.

[7] M.F. Abrar, S. Rehman, N. Rashid and S. Ali, "Large Scale Agile Adoption Model from Management Perspective," International Journal of Computer Applications, vol. 152, no. 2, pp. 31.35, 2016.

[8] M. Lindvall, D. Muthig, A. Dagnino, C. Wallin, M. Stupperich, D. Kiefer, J. May and T. Kahkonen, "Agile software development in large organizations," Computer, vol. 37, no. 12, pp. 26-34, 2004.

[9] J. Barlow, J. Giboney, M. Keith, D. Wilson, R. Schuetzler, P. Lowry and A. Vance, "Overview and guidance on agile development in large organizations," Communications of the Association for Information Systems, vol. 29, no. 2, pp. 25-44, 2011.

[10] B. Boehm and R. Turner, "Management challenges to implementing agile processes in traditional development organizations," IEEE Software, vol. 22, pp. 30-39, 2005.

[11] Ö. Uludag, M. Kleehaus, C. Caprano and F. Matthes, "Identifying and Structuring Challenges in Large-Scale Agile Development Based on a Structured Literature Review," in Proce. The 22nd International Conference on Enterprise Distributed Object Computing, Stockholm, Sweden, 2018, pp 191-197.

[12] B. Hobbs and Y. Petit, "Agile Methods on Large Projects in Large Organizations," Project Management Journal, vol. 48, no. 3, pp. 3-19, 2017.

[13] T. Dingsøyr and D. P. K. Falessi, “Agile Development at Scale: The Next Frontier," IEEE Software, 2019, pp. 1-10.

[14] T. Karvonen, H. Sharp and L. Barroca, "Enterprise Agility: Why Is Transformation so Hard?," in Proceedings The 19th International Conference on Agile Processes in Software Engineering and Extreme Programming, Porto, Portugal, 2018, pp. 131-145.

[15] R. Duijs, P. Ravesteyn and S. Marlies, "Adaptation of enterprise architecture efforts to an agile environment," in Proc. The 31ST Bled eConference Digital Transformation: Meeting the Challenges, Blend, Slovenia, 2018, pp. 389-400.

[16] A. Gill, "Adaptive enterprise architecture driven agile development," in Proc. The International Conference on Information Systems Development, Harbin, China, 2015, pp. $1-9$.

[17] J. Persson, J. Nørbjerg and P. Nielsen, "Improving ISD Agility in Fast-Moving Software Organizations," in Proc. The European Conference on Information Systems, Istanbul, Turkey, 2016. 